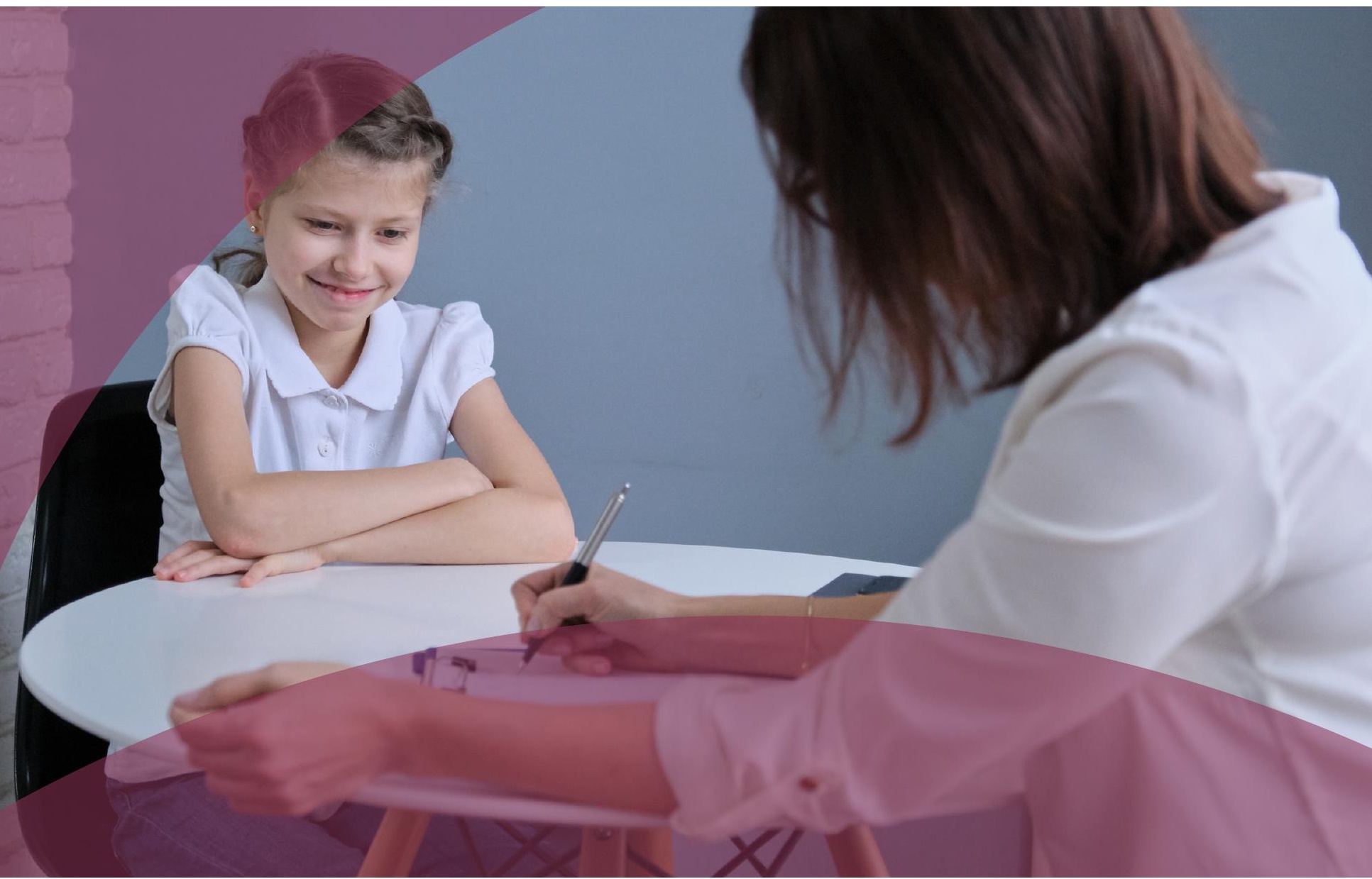

\title{
Do autistic girls talk differently about social groups?
}

New data, published in the Journal of Child Psychology and Psychiatry, suggest that pronoun use during natural conversation might inform us about clinically meaningful social function. Amber Song and colleagues set out to understand whether they could identify natural language markers of social characteristics exhibited by girls and boys with autism. To do so, they recruited 50 schoolaged autistic children (17 girls and 33 boys who were matched for level of social impairment) and 37 typically developing children who were matched for age (mean age $=11.35$ years) and IQ (mean IQ = 107). They then asked the children to engage in a

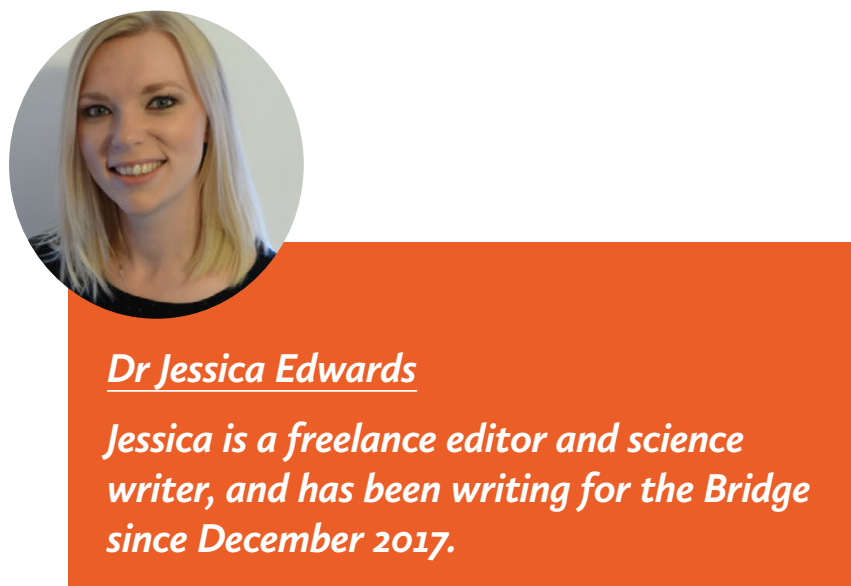


short, informal conversation. From the conversation sample, the researchers analysed the use of first person (e.g., "we" and "us") and third person (e.g., "they" and "them") plural pronouns to determine whether autistic girls and boys talk differently about social groups.

Overall, autistic children used significantly fewer plural pronouns than their typically developing peers, indicating that they talked less about social groups. However, autistic girls talked more about social groups than autistic boys. Yet compared to typically developing girls, autistic girls talked more about groups they were not a part of; this was indicated by more frequent use of the pronouns "they" and "them" and less frequent use of "we" and "us". The researchers speculate that this finding might demonstrate potential awareness of social exclusion in affected girls.

Finally, Song et al. tested whether pronoun use could predict ADOS-2 scores in children with autism. The ADOS-2 consists of semi-structured tasks that involve communication, social interaction, and play to assess different aspects of autism. After controlling for age and IQ, the researchers found that the conversation sample "we" and "they" variants significantly predicted ADOS-2 social affect but not repetitive behaviour scores in girls. Thus, pronoun use might predict individual differences in social function in autistic girls.

"This finding is especially important because girls with autism are not as well understood as boys", explains study author Julia Parish-Morris. "When we learn more about how girls with autism behave in the real world, it helps us develop personalized supports to address their unique needs most effectively". Although this study was relatively small, the researchers believe the data serve as a proof-of-concept that natural conversation samples might inform us about clinically meaningful social similarities and differences between girls and boys with autism.

Song et al. now plan to expand their work to include more participants and to collect data using semistructured question-and-answer formats. From here, they hope to determine whether the identified patterns hold true for other age groups and those with lower, or higher, IQ scores. Future research might then test whether subtle linguistic clues can help improve identification of autism in girls.

\section{This finding} is especially important because girls with autism are not as well understood as boys

\author{
Julia Parish-Morris
}

\section{Referring to:}

Song, A. et al. (2020), Natural language

markers of social phenotype in girls with

autism. J. Child Psychol. Psychiatr. doi:

10.1111/jcpp.13348.

洋 ACAMH CAMHS around the

1 Campfire: Trajectories of autistic social traits in CYP and disordered eating at age 14 years

"̈im ACAMH Webinar for parents:

2 Tackling low diagnosis for autism in girls, A Ozsivadjian \& M Murin,

滴 JCPP Video Abstract: Anorexia

nervosa and autism: a prospective twin cohort study, L Dinkler

To visit any of the links related to this article, click here to go to the ACAMH website. 